\title{
Turbulent Oscillating Channel Flow Subjected to Wind Stress
}

\author{
W. Kramer ${ }^{1}$, H.J.H. Clercx ${ }^{1}$, and V. Armenio ${ }^{2}$
}

1 Fluid Dynamics Laboratory, Department of Physics, Eindhoven University of Technology, P.O. Box 513, 5600 MB Eindhoven, The Netherlands, w.kramer@tue.nl; h.j.h.clercx@tue.nl

2 V. Dipartimento di Ingegneria Civile e Ambientale, Università degli studi di Trieste, Trieste, Italy, armenio@dica.units.it

\begin{abstract}
The channel flow subjected to a wind stress at the free surface and an oscillating pressure gradient is investigated using large-eddy simulations (LES). A slowly pulsating mean flow occurs, with the turbulent mechanics essentially being quasi steady. Logarithmic boundary layers are present at both the bottom wall and the free-surface. Turbulent streaks are observed in the bottom and free-surface layer. The mean velocity and the structures emerging in the flow have been discussed.
\end{abstract}

\section{Introduction}

In addition to tidal forcing, the wind stress exerted on the free surface might play a role in driving the flow in estuary regions. Therefore, we estimate the amplitude of both effects based on data for the Westerschelde estuary. Here, the flow is strongly tidal driven with a typical fluid velocity during high tide of $U=0.2-1.0 \mathrm{~m} / \mathrm{s}$. The Reynolds number based on the depth $(h=10 \mathrm{~m})$ is $\operatorname{Re}_{h}=U h / \nu=10^{6}-10^{7}$ with $\nu$ the kinematic viscosity. For this range of $\operatorname{Re}_{h}$ the flow is turbulent for all phases of the tidal cycle. The estimated maximum wall stress due to the tides is $0.08-1 \mathrm{~N} / \mathrm{m}^{2}$. A typical wind velocity of $7 \mathrm{~m} / \mathrm{s}$ yields a wind stress of $0.17 \mathrm{~N} / \mathrm{m}^{2}$. The wind stress and the wall stress are thus of the same order. The turbulent flow in the Westerschelde estuary is characterized by a large Keulegan-Carpenter number, $\mathrm{KC}=100-600$, which indicates that the time scale related to the turbulence is much shorter than the tidal period.

Turbulence is known to accelerate mixing and transport of particles. Hence, it is of relevance to sand sedimentation and dispersion of plankton formations. This work is part of a project aimed at modeling dispersion of plankton in geophysical flows. The approach is to first investigate particle dispersion at the smallest turbulent scales. This knowledge can then be used for modeling dispersion statistics in large-scale geophysical flows. 
To inquire the type of turbulence in such a geophysical flow we performed large-eddy simulations of an oscillating flow subjected to a wind stress at the free surface.

\section{Problem description}

In this work, we study the turbulent oscillating channel flow subjected to a wind stress by means of large eddy simulations (LES). The largest structures in the flow are obtained solving the filtered Navier-Stokes equation,

$$
\frac{\partial \widehat{u}_{i}}{\partial t}+\frac{\partial \widehat{u}_{i} \widehat{u}_{j}}{\partial x_{j}}=-\rho^{-1} \frac{\partial \widehat{p}}{\partial x_{i}}+\nu \frac{\partial^{2} \widehat{u}_{i}}{\partial x_{j} \partial x_{j}}+\widehat{f}_{i}-\frac{\partial \tau_{i j}}{\partial x_{j}} .
$$

Here, $\widehat{u}_{i}, \widehat{p}$ and $\widehat{f}_{i}$ are the filtered velocity, the filtered pressure and the filtered external forces, respectively. The density $\rho$ is assumed to be constant and, hence, the velocity field is divergence free. The stresses $\tau_{i j}$ the small scales of the flow exert on the large scales are modeled using a dynamic eddy-viscosity model combined with a scale-similarity model [1].

The channel domain is periodic along the horizontal $x$ - and $y$-direction and is bounded in the vertical direction by a no-slip bottom $(z=0)$ and a free-surface layer at the top $(z=1)$. The horizontal dimensions are larger than the channel height to capture the largest eddies in the domain $\left(l_{x} \times\right.$ $\left.l_{y} \times h=2 \times 1.4 \times 1\right)$. To mimic a tidal flow an oscillating pressure gradient $\widehat{f}_{p}=-U \omega \cos \omega t$ with frequency $\omega=1 / 80$ and velocity amplitude $U=1$ is applied over the $x$-direction. Along the positive $x$-direction a constant wind stress $\tau_{\text {wind }}=10^{-3}$ acts on the free-surface layer. All quantities are made dimensionless using the height of the channel and the velocity amplitude of the tidal oscillation.

Equation (1) is solved using a finite-volume method based on the method by Zang et al. [8]. The Reynolds number and Keulegan-Carpenter number are decreased to $\operatorname{Re}_{h}=U h / \nu=5 \times 10^{4}$ and $\mathrm{KC}=80$ to make the simulations feasible. As no wall-model is available for this kind of flow, the wall stress must be resolved by the LES. For the no-slip boundary layer to be resolved the first grid cell is set to be equal to one wall unit, which is defined as $\Delta z^{+}=\Delta z / z^{*}$ with $z^{*}=\nu / u_{\tau}$ and $u_{\tau}=\sqrt{\tau_{w, \max } / \rho}$. The maximum wall stress $\tau_{w, \max }$ can be estimated in advance using the maximum wall stress for the purely oscillating case and adding the surface stress. Using data from Jensen et al. [3] this yields an estimated maximum of $\tau_{w, \max }=3.0 \times 10^{-3}$. Resolving the free-surface layer proved to require a finer resolution. Here, the first grid cell height is $\Delta z^{+}=1 / 2$. The horizontal grid spacing required in the boundary layer for resolved LES is $\Delta x^{+} \approx 60$ for the streamwise direction and $\Delta y^{+} \approx 30$ for the spanwise direction. These requirements are reached with a resolution of $48 \times 64 \times 128$ if grid stretching is applied for the vertical. For these grid resolutions the numerical model has been used successfully to simulate a turbulent oscillating channel flow as is typical for the Gulf of Trieste [5]. 


\section{Mean velocity}

The obtained flow field is strongly turbulent during the complete cycle. The combination of the tidal and wind forcing results in a pulsating mean flow in the $x$-direction (Fig. 1). The mean flow is obtained by plane and phase averaging. The wind stress drives a constant mean flow in the interior with strong shear layers at the bottom and free-surface layer. A similar velocity profile is observed, although with a larger velocity, when the oscillating pressure gradient is absent. The pressure gradient will accelerate and decelerate the flow in positive $x$-direction in the first half period. While in the second half period it will accelerate and decelerate the fluid in the negative $x$-direction. The mean velocity reaches a maximum velocity of about $U_{\max }=1.4$ in the interior at $\varphi=100^{\circ}$. The flow then decreases, subsequently reverses and reaches a value of $U_{\max }=-0.6$ at $\varphi=250^{\circ}$.

Investigation of the bottom boundary layer reveals that a clear log layer, $u^{+}=\kappa^{-1} \log z^{+}+C$, is present with $\kappa=0.41$ and the constant $C$ ranging between 5 and 7 for the phases $30-150^{\circ}$. The same increased values of $C$ were found by Salon et al. [5] for the purely oscillating case. For the phases, when the mean velocity is either small or reversing, no log layer is present. The wind stress, which is no opposite to the wall stress, disturbs the formation of a bottom $\log$ layer for $210-240^{\circ}$. When the wall stress amplitude reaches a maximum again at the phases $270^{\circ}$ a log layer reappears. A thinner 'viscous sublayer' for $\langle u\rangle-\langle u\rangle_{z=h}$ is observed at the free surface. Tsai et al. [7] argued that presence of horizontal fluctuations at the free-surface has a similar effect as surface roughness, which leads to a decrease of the viscous sublayer. Starting

$0^{\circ}-60^{\circ}$

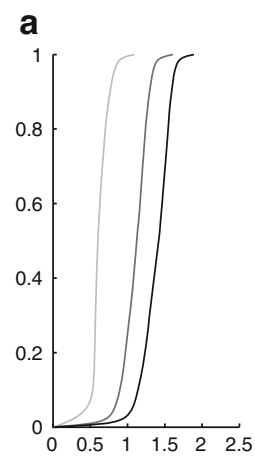

$\langle u\rangle$

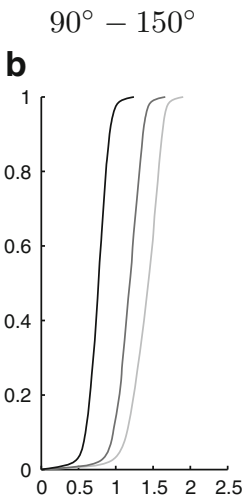

$\langle u\rangle$ $180^{\circ}-240^{\circ}$

C

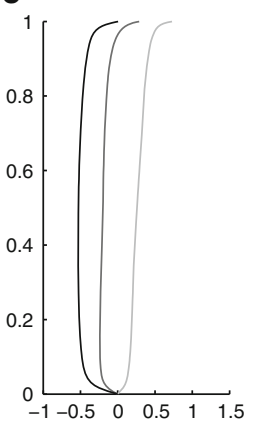

$270^{\circ}-330^{\circ}$

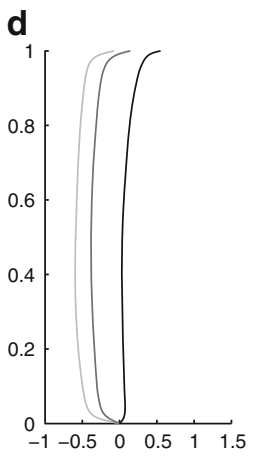

Fig. 1. The mean streamwise velocity for oscillating channel flow subjected to a wind stress for different phases (a) $0-60^{\circ}$, (b) $90-150^{\circ}$, (c) $180-240^{\circ}$ and (d) $270-330^{\circ}$. The darker colors represent the later phases, e.g., in (a) the profiles are light grey, dark grey and black for $0^{\circ}, 30^{\circ}$ and $60^{\circ}$, respectively. 

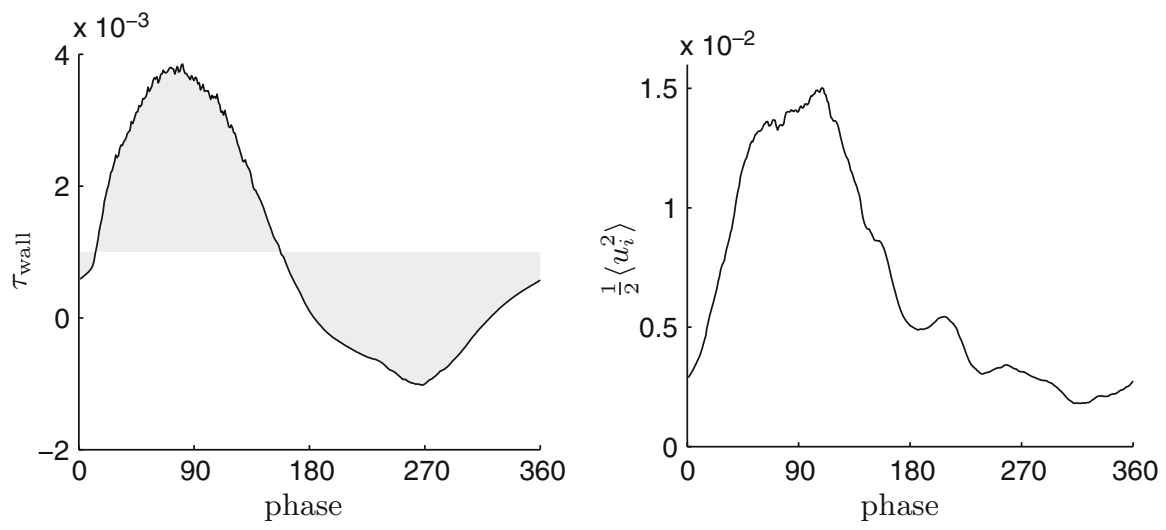

Fig. 2. The phase averaged wall stress (left) and the turbulent kinetic energy (right). Integrated over a period the wall stress matches the wind stress, $\tau_{\text {wind }}=1 \times 10^{-3}$.

at $210^{\circ}$ the thickness of the viscous layer seems to increase to $\Delta z^{+}=10$ pushing the log layer further away from the free surface.

The wall stress is given in Fig. 2. Integrated over a period the wall stress matches the applied wind stress. The typical Reynolds number $\operatorname{Re}_{\omega}=$ $U_{\max }^{2} / \omega \nu=4 \times 10^{6}$ in the first half cycle and $7 \times 10^{5}$ in the second half cycle. The wall stress profiles in the first and second half cycle are in agreement with the profiles Jensen et al. [3] observed for different $\operatorname{Re}_{\omega}$ (see their Fig. 9). Now following their observations, the Reynolds numbers indicate that the first half cycle is in the fully turbulent regime leading to an increased wall stress and increased levels of turbulence. The second half cycle is in the intermittent turbulent regime. The small increase of the wall stress amplitude and turbulent kinetic energy around $\varphi=270^{\circ}$ is most likely due to a burst like production of turbulence occurring in the deceleration phase, as was observed by Hino et al. [2].

\section{Structures of the turbulent flow}

The varying wall stress has an impact on the intensity and structure of turbulence. The turbulent structures are visualized using the fluctuations in the streamwise velocity. In the first half cycle the strong shear causes the formation of turbulent streaks in the bottom layer (Fig. 3). When the flow decelerates the streaks are smoothened by viscosity. Then in the second half cycle wall stress builds up again and turbulent streaks reappear at $\varphi=270^{\circ}$. The observed streak spacing is identical to the typical spacing in canonical wall turbulence. The streaks at the free surface have similar spacing but some distinct low-speed spots are present throughout the cycle. These features were also observed by Tsai et al. [7] for a stress-driven free-surface flow. 


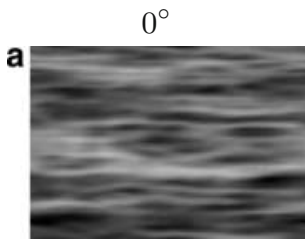

$0^{\circ}$

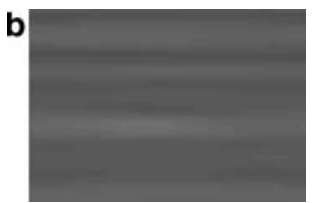

$90^{\circ}$

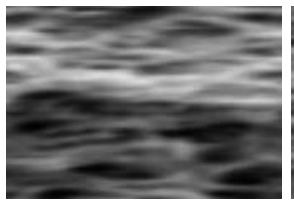

free-surface layer

$90^{\circ}$

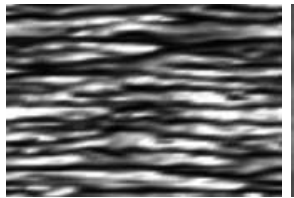

bottom layer $180^{\circ}$

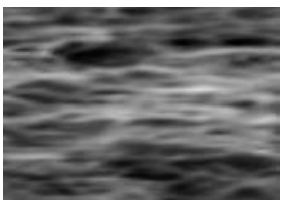

$180^{\circ}$

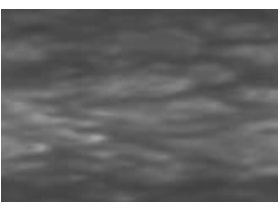

$270^{\circ}$

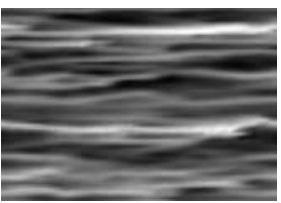

$270^{\circ}$

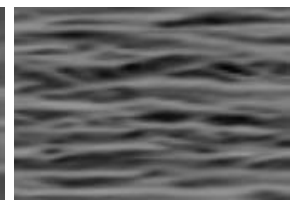

Fig. 3. Gray-scale plots of the streamwise velocity fluctuations (a) at the free surface $(z=1)$ and $(\mathbf{b})$ in the bottom boundary layer $\left(z=10^{-2}\right)$. Values are ranging between -0.3 (black) and 0.3 (white).

To study the turbulent structure in the interior we constructed Lumley triangle or turbulence triangle (Fig. 4). In each horizontal plane the second and third invariants, $\mathbb{I}_{b}$ and $\mathbb{I}_{b}$, respectively, of the anisotropic Reynolds stress tensor, $b_{i j}=\left\langle u_{i}^{\prime} u_{j}^{\prime}\right\rangle /\left\langle u_{k}^{\prime} u_{k}^{\prime}\right\rangle-\delta_{i j} / 3$, are calculated. The combination of the two invariants relate to an anisotropic state of the Reynolds stresses. For most phases we observe one-component turbulent structures in the bottom boundary layer, where the streamwise fluctuations are dominant over the spanwise and vertical fluctuations. The structures become more 3D isotropic away from the wall. This specific signature in the Lumley map, compares well with the one observed for steady boundary layer flow [4]. In the free-surface layer turbulence is also nearly one component throughout the cycle. The laminarization of the flow in the bottom boundary layer in the deceleration phases leads to $2 \mathrm{D}$ Reynolds stresses or pancake-like structures at $180^{\circ}$. The region where two-component turbulence is observed then further protrudes into the domain at $240^{\circ}$. In the wall layer a return to one-component turbulence is driven by the increasing amplitude of the wall stress.

\section{Conclusion}

The oscillating pressure gradient together with a fixed stress at the free surface in the same direction results in a pulsating flow. The first half cycle is in the turbulent regime with a strongly increased wall stress. Due to the decreased mean velocity in the second half cycle turbulence falls back to the intermediate regime with a burst-like production of turbulence in the deceleration phase. However, in real estuary flows it can be expected that both half cycles are in the turbulent regime because of the overall higher Reynolds number. 

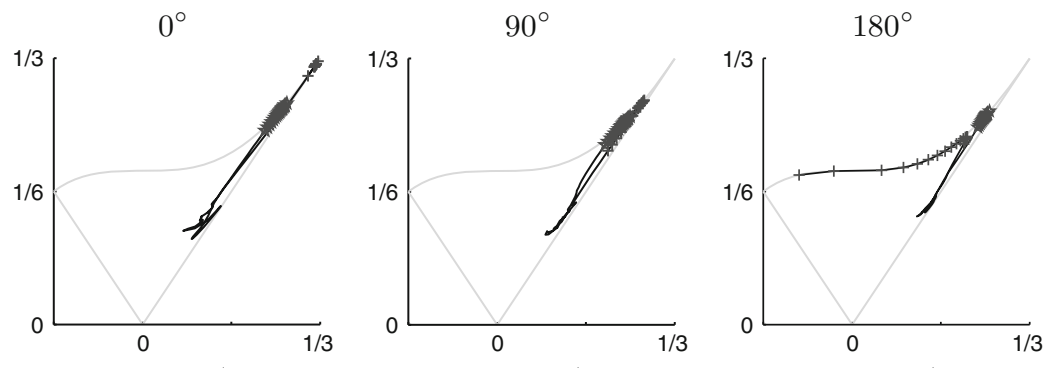

$\left(\mathrm{III}_{b} / 2\right)^{1 / 3}$

$\left(\mathrm{III}_{b} / 2\right)^{1 / 3}$

$\left(\mathrm{III}_{b} / 2\right)^{1 / 3}$

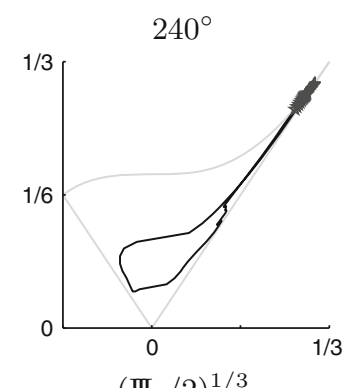

$\left(\mathrm{III}_{b} / 2\right)^{1 / 3}$

Fig. 4. The anisotropy of the Reynolds stresses is mapped to the turbulence triangle (grey). The lower edge $(0,0)$ relates to $3 \mathrm{D}$ isotropic structures, the left edge $(-1 / 6,1 / 6)$ to $2 \mathrm{D}$ isotropic structures and the right edge $(1 / 3,1 / 3)$ to $1 \mathrm{D}$ structures. The black line quantifies the structure of the turbulence at different depths within the fluid column. Marked are the first twenty grid points above the bottom wall (plus) and below the free surface (star).

At the bottom a logarithmic layer with strong turbulent streaks develops in the acceleration phases. In the deceleration phase a relaminarization takes place as the streaks are smoothened by viscosity. High-speed streaks are present at the free surface throughout the cycle. The observed pattern is in agreement with the findings of Tsai et al. [7]. Shear production leads to intense streamwise fluctuations in the boundary layer. Spanwise and vertical fluctuations are less intense and overall one-component turbulence is observed. The relaminarization of the bottom boundary layer gives rise to two-component turbulence that protrudes into the interior.

The large Keulegan-Carpenter number results in turbulent flow where the turbulence can be considered quasi-stationary for most phases. This is confirmed by the presence of log layers during acceleration, which are related to steady boundary-layer turbulence. This steady-state behavior is in agreement with the observations by Scotti and Piomelli [6] for pulsating flows.

In future work we will investigate the effect of wind stress orientation and density stratification. A stable stratification can suppress turbulent motion in the vertical direction. This might decouple the interaction between the bottom and free-surface layer turbulence. 


\section{References}

1. Armenio V, Piomelli U (2000) Flow Turb Comb 65:51-81

2. Hino M, Kashiwayanagi M, Nakayama A, Hara T (1983) J Fluid Mech 131: 363-400

3. Jensen BL, Summer BM, Fresoe J (1989) J Fluid Mech 206:265-297

4. Kim J, Moin P, Moser R (1987) J Fluid Mech 177:133-166

5. Salon S, Armenio V, Crise A (2007) J Fluid Mech 570:253-296

6. Scotti A, Piomelli U (2001). Phys Fluids 13:1367-1383

7. Tsai WT, Chen SM, Moeng CH (2005) J Fluid Mech 546:163-192

8. Zang Y, Street RL, Koseff JR (1994) J Comp Phys 114:18-33 